\title{
MICOSE DE LOBO
}

\author{
Domingos Silva*:
}

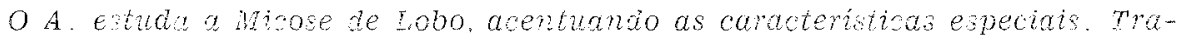

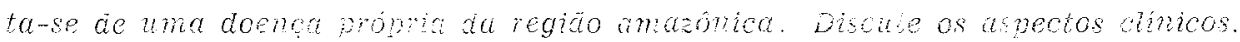

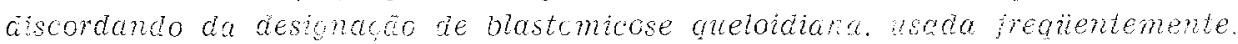
pois em sua tasta casioten no Devartanento de Dematologia do Centro Bio Médico da Univervidade Federal do Para foi possivel distinguir 5 formas chinicas

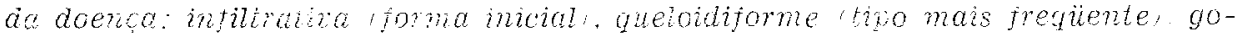

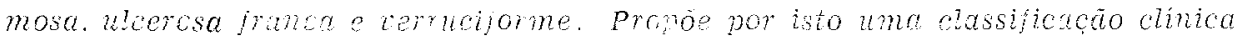
da Micose.
\end{abstract}

\section{SINONIMIA}

- Micose de Jorge Lcbo

- Blastomicose queloidiana

- Blastomiccse queloidifcrme

\section{IMPORTANCIA DO TEMA}

A Micose de Lobo constitue, sem nenhuma dúvida. um dos mais fascinantes temas de Patologia Tropical.

Seja por sua relativa raridade, de vez que só estão registrados na literatura médica 85 casos: seja por suas características clínicas, que a diferenciam das outras parablastomicoses, ou ainda, pelas intermináveis discussōes quanto à posição do agente responsável na Sistemática Micológica, pode-se afirmar, sem receio de contestação, que os vários aspectos desta micose continuam sujeitos a sérias divergências, entre os numerosos pesquisadores que têm tratado do assunto.
Por esta razão. julgamos oportuno tracar un perfil da Miccse de Lobo, abordando alguns angulos que nos parecem controvertidos

\section{EPIDEMIOLOGIA}

a) A micose tem sido encontrada somente na regiāo neotrópica. Por longo tempo considerada exclusiva da regiāo amazonica, fci, pouco a pouco, sendo identificada em cutras áreas das Américas do Sul e Central:

Caso 10 - em Costa Rica -- por Romero \& Trejos (20).

Caso 15 - no Fanamá … por Herrera 112 ,

Caso 19 - na Venezuela - por Campo Aachen (6).

Caso 26 - na Venezuela - por Convit \& Escuder (7).

Caso 27 - na Colômbia - por Henao Pelayo (11).

* Trabalho do Departamento de Dermatologia Centro Biomédico. Universidade Federal do Pará.

* Professor Titular de Dermatologia.

Trabalho apresentado ao VII Congresso da Scciedade Brasileira de Medicina Tropical. Manaus, Ieva. reiro de 1971

Recebldo para publicação em 30-1-1972. 
Caso 28 - na Guiana Francesa - por Fontana (9)

Caso 31 - na Venezuela - por Reyes, Goihman \& Goldstcin 1191.

Casos 32/33 - na Guiana Francesa por Silverie \& Cols. 1251.

Cases 42 a $48^{\circ}$ - no Surinam - por Wiersema \& Niemel 127 .

$$
\text { Caso } 59 \text { - na Colômbia - por Ville- }
$$
gas $(26)$

Casos 69/61 - na Venezuela -- por Battistini \& Cols. (4).

Caso 77 - na Guiana Francesa - por Pradinaud \& Cols. 181 .

Temos assim 20 casos fora do Brasil, correspozdendo a $23,6 \%$ dos registros conhecidos

Ultimamente. algumas observaçoes brasileiras têm sido anotadas em Mato Grosso e Goiás, tais como a de Barbosa \& Doles (2) e entre os indios caiabis, por Machado (16) e Baruzzi \& Cols. (3), que na realidade devem ser incluidas na área geográfica da chamada Amazonia Legal.

Assim, com raras exceçōes tcasos $10 \mathrm{e}$ 15). todos os demais foram registrados na Amazonia (Brasil) ou em paises vizinhos. limitrofes àquela área (Venezuela, Guiana Francesa, Surinam e Colombial, pertencentes ao mesmo complexo biogeográfico. com as mesmas caracteristicas fisicas e climáticas: matas densas, entremeiadas de rios e igapós, clima quente e úmido, e alta pluviosidade anual

b) Dos 85 casos conhecidos até agora, 65 estāo situados no Brasil e praticamente todos adquiriram a micose na regiāo amazonica. Dos 20 casos registrados fora do Brasil, 18 o foram em paises vizinhos ácuela área -... 7 no Surinam, 5 na Verezuela, 4 na Guiana Francesa e 2 na Colómbia.

c) A Micose não parece ser tāo rara na Amazônia brasileira. Morais (17) afirina que esta doença é a micose profunda mais frequente no Estado do Amazonas. No Estado do Pará, segundo nossos registros e de outros Departamentos da Faculdade de Medicina da Universidade Federal do Pará, foi possivel identificar 29 casos, 19 nossos $(8,15,21.28,23$ e 24), 8 de Mon- teiro Leite $(13,14)$ e 2 de Azevedo (1) - o que representa $34.5 \%$ dos casos conhecidos

(i) A Micose atinge quase que exclusivamente individuos engajados em trabatho ce lavoura ou que permaneceram longos perídos de tempo em zonas florestais.

Provavelmente o fungo vive na natureza, em estado saprofítico. Os pacientes referem com muita freqüencia, como inicio de sua história clínica, a existência de traumatismos com fragmentos de vegetais ou a mordedura de artropodos, o que nos levaria a correlacioná-los com o inóculo.

e) Este fato talvez explique a acentuada freqüencia com que as lesōes se localizam nas orelhas. Muitas vezes os pacientes informam que a micose se instalou em lccais onde sofreram a picada de artrópodos. A nosso pedido, dois doentes trouxeram alguns exemplares de um carrapato (Ixcaidae), inafortunadamente mortos há muito tempo e já excicados. As tentativas que fizemos com triturados dos mesmos incculaçōes e culturası restaram sempre negativas, o aue talvez tenha ocorrido na dependência do estado do material. Pretendemos continuar as investigações nesta área

Borelli (5) acredita que a preferência de localizaçāo das lesōes no pavilhão auricular esteja condicionada a certa oligotermofilia do cogumelo. A este respeito. Monteiro Leite rop. cit.) anotou um fato interessante: "os nodulos têm temperatura nitidamente inferior a normal, o que coservamos em todos os 3 casos, o que nos chamcu ncssa atencão no decurso do exame clínico, dando assim uma sensacāo contrária a que faria supor o asfecto das lesões.. As nossas investigacoes, em alouns pacientes, revelaram abaixamento de temperatura nas lesões ao nivel das orelhas $c$ mesmo não acontecendo nas lesōes situadas em outras regióes. As temperaturas axilar, cral ou anal dos pacientes mantiveram-se sempre nos limiares da normalidade.

f) A localização nas orelnas corresponde a $25,9 \%$ ( 22 casos). percentagen que se elevará se excluirmos 8 casos da estatistica em que não nos foi possivel obter dados concretos. 
g) Por ordem de frequência, em 77 casos onde foi possivel classificar corretamente a distribuicão das lesões, obtivemos os seguintes dados:

\section{Localização}

orelhas.

membros inferiores ........... 17

No de casos

membros superiores ............ 12

disseminadas (diversas regióes) .... 12

face . ................... 7

sacra/glútea ............... 3

tórax . ................. 2

pescoco . ................ 1

cmbro

h) Admite-se que o fungo, vivendo na natureza como saprófita, seja inoculado através efracöes tegumentares, no decurso das atividades de lavoura. Este mecanismo é perfeitamente explicado, e. g.. na crcmoblastomicose, na qual as lesões se localizam preferencialmente nos nembros inferiores. Já na Micose de Lcbo, onde os indivíduos expostos a doença sāo do mesmo grupo profissional -- lavradores, há que acentuar a preferencia pela localizacão nas orelhas, o oue talvez permita-nos enfatizar o papel de certos artrópodos na veiculação do material infectante.

i) Un fato paradoxal a respeito da Miccse de Lobo ê que as lesōes são extremamente ricas em parasitos, contrastando com a tendência em permanecerem localizadas curante muito tempo. É possivel que - fenomeno esteja correlacionado à baixa patogenicidade do fungo, ou ao fato de. no dechrso de sua muitiplicacác, as gêmulas filhas só se destacarem quando atingem a iciade aduita e então seu diâmetro é maior que a luz dos vasos. É frequiente observar parastos eni cadeias de 3.4 e até 7 elementos formas catenulares,

j) Nảo obstante há que assinalar formas disseminadas da micose, fazendo crer numa disseminacäo hematogénica, dadas as caracteristicas clinicas apresentadas por esses doentes. Reforcando este argumento, Fraga \& Miranda (10) registraram um caso em que os cortes histopatológicos davam idéia da existência de parasitos no interior dos vasos sanguíneos, sugerindo a possibilidade de disseminação hematogênica.

\section{CLINICA}

Esta Micose apresenta diversos tipos clínicos. O conceito generalizado de que a Micose de Lobo se caracteriza por certo monomorfismo, traduzido pela presenca de lesōes semelhantes a queloides. dai a denominaçāo de blastomicose queloidiana ou blastomicose quelcidiforme, tem que ser reformulado. Na maioria dos casos, com efeito, as lesōes obedecem a esse tipo semiótico. É preciso acentuar porém, que as lesões podem revestir outros tipos clínicos. $\mathrm{Na}$ nossa experiencia, face a um grande número de casos que estudanos. foi possível identificar formas outras, o que nos levou ao esboço de uma classificacão, baseada simplesmente no aspecto semiótico das lesões:

\section{Formas clinicas \\ 1. Infiltrativa \\ 2. Queloidifome: \\ 3. Gomosa: \\ 4. Ulcerada; \\ 5. Verruciforme.}

À base da documentaçāo que apresentamos ficam bem esclarecidos os diversos tipos acima

Algumas destas formas se embricam evidentemente. Assim. os tipos gomoso e vicercso, sendo este, em algins casos. fase evolutiva do primeiro. Outras vezes, a lesảo ulcerada é um fenomeno transitorio no quadro clinico. havendo cicatrizacāo fácil. tal como acontece no tipo queloidiforne, oue pode apresentar ulceraçoes passageiras, muitas vezes cicatrizando espontareamente.

Entretanto, há que relatar cascs que semicticamente dovem sor ercarados como francamente ulcerosos, permanecendo como tal durante longo tempo.

o tipo infiltrativo parece ser a forma inicial da micose

O tipo veriuciforme fui bem evidenciado por Pradinand. Joly, Basset. Basset do Grosshans lop. cit.), que enfatizaram inclusive o aspecto em tela.

Tendo em vista que o aspecto queloidial não é o exclusivo nesta doença, preferimos a designacão de Micose de Lobo 


\begin{tabular}{|c|c|c|c|c|}
\hline $\begin{array}{l}\text { CASO } \\
\text { No }\end{array}$ & $\begin{array}{c}\text { PACIEN- } \\
\text { TES }\end{array}$ & SEXO & LOCALIZAC̣̃̃O & ORIGEM DO CAS \\
\hline & $\cdots \cdot \cdots$ & & & \\
\hline 1 & J. B. & M & Regiāo sacra & Armazônia -.. Brasil \\
\hline 2 & J. D. J. & M & orelha direita & -. - Brasil \\
\hline 3 & J. L. C. & M & Antebraco direito & Amazônia … Brasil \\
\hline 4 & & M & Antebraço direito & Amazônia Brasil \\
\hline 5 & F. V. M. & M & Pernas & Amazonia -.. Brasil \\
\hline 6 & V. B. M. & M & Perna D/Reg. Glutea & Amazônia … Brasil \\
\hline 7 & H. I. & M & Perna D. & Amazonia -. Brasil \\
\hline 8 & S. R. S. & M & Orelha E. & Amazônia -... Brasí \\
\hline 9 & D. $\mathrm{O}$. & M & Braço E. & Amazônia - Brasil \\
\hline 10 & & $\mathrm{M}$ & Pernas & COSTA RICA \\
\hline 11 & F. J.S. & $\mathbf{F}$ & Perna E & Amazônia - Brasil \\
\hline 12 & O. B. S. & M & Orelha E. & Amazōnia -- Brasil \\
\hline 13 & F. S. B. & M & Orelha $\mathrm{D}$. & Amazônia -- Brasil \\
\hline 14 & A. M. C. & $\mathbf{F}$ & Nariz & Amazônia - Brasil \\
\hline 15 & & M & Perna D. & PANAMÁ \\
\hline 16 & & M & - & Amazônia -.. Brasil \\
\hline 17 & A. J. O. & M & Disseminado & Amazônia -.- Brasil \\
\hline
\end{tabular}

Jorge Lobo

Paulo Azevedo

Eaulo Anevedo

A. Trejos \& A. Romero

i. S Carineiro 1952

J. Monteiro Leite 1954

J. Monteiro Leito 1954

;. Monteiro Leito 1954

J. M. Heriera 1955

C. S. Lacaz \& Cols. 1955

Domingos Silva 1956 
MICOSE DE L OBO

$1931 \ldots 1971$

\begin{tabular}{|c|c|c|c|c|}
\hline $\begin{array}{c}\text { CASO } \\
\text { No }\end{array}$ & $\begin{array}{c}\text { PACIEN- } \\
\text { TES }\end{array}$ & SEXO & LOCALIZACAOO & ORIGEM DO CASO \\
\hline 18 & R. N. C. & M & Orclha E. & Amazônia … Brasil. \\
\hline 19 & S. G. M. & M & Orelha $\mathrm{E}$ & VENEZIJELA \\
\hline 20 & V.F.S. & M & Nariz & Amazônia -- Brasil \\
\hline 21 & A. P. I. & $\mathrm{M}$ & Orelha E. & Amazônia $\quad$ Brasil \\
\hline 22 & Coa & M & Bracos, pernas c dorso & Mato Grosso -- Brasil \\
\hline 23 & Acuaxi & $\mathrm{M}$ & Perna e braço D. & Mato Grosso - Brasil \\
\hline 24 & P. G. M. & M & Orelha is. & Anazônia - Brasil \\
\hline 25 & C. M. & M & Crelha $\mathrm{E}$ & Amazōnia - Brasil \\
\hline 26 & & M & Crclha D. & VENEZUELA \\
\hline 27 & & M & & COLÔMBIA \\
\hline 28 & & & & GUIANA FRANCESA \\
\hline 29 & P. S. M. & M & Região peitoral & Amazônia -- Brasil \\
\hline 30 & B. M. S. T. & $\mathbf{F}$ & Orelha $\mathrm{E}$ & Amazônia … Brasil \\
\hline 31 & J. P. & M & Disseminado & VFNEZUELA \\
\hline 32 & & & & GUIANA FRANCESA \\
\hline 33 & & & & GUIANA FRANCESA \\
\hline 34 & R. P. & M & Perna E. & Amazônia - Brasil \\
\hline
\end{tabular}

AUTORES

ANOS

R. D. Azulay, J. Miranda, J. D. Azulay : 1957

I. Campo - Aachen 1957

J. Teixeira 1957

J. Teixeira : 1957

M. J. Pereira Filho 1957

M. J. Pereira Filho 1957

F. Fialho 1958

F. Fialho 1958

J. Convit \& J. Escuder 1958

C. Henao Pelayo 1958

R. Fontan 1960

Domingos Silva \& Cols. ' 1961

Domingos Silva \& Cols $\quad 1961$

O. Reys, M. Goihman \& C. Golcstein 1961

C. R. Silverie, P. Rarvisse, J. P. Vilar \& C. Moulins 1962

C. R. Silverie, P. Rarvisse, J. P. Vilar \& C. Moulins 1962

M. Morais : 1962 
$1931-1971$

\begin{tabular}{|c|c|c|c|c|}
\hline $\begin{array}{c}\text { CASO } \\
\text { No }\end{array}$ & $\begin{array}{l}\text { PACIEN- } \\
\text { TES }\end{array}$ & SEXO & LOCALIZAC̣ÃO & ORIGEM DO CASO \\
\hline & 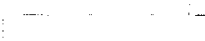 & $\ldots$ & & $\ldots$ \\
\hline 35 & R. M. M. & M & Orelha E. & Amazônia - Brasil \\
\hline 36 & R. R. N. & M & $\begin{array}{l}\text { Perna } \mathrm{D} \text {, antebraco e } \\
\text { cotovelo } \mathrm{D} \text {. }\end{array}$ & Amazônia - Brasil \\
\hline 37 & W. P. P. & M & Orelha D. & Amazònia - Brasil \\
\hline 38 & M. P. R. C. & $F$ & Perna D. & Amazônia - Brasil \\
\hline 39 & J. B. & M & Glútea $\mathrm{E}$. & Amazónia - Brasil \\
\hline 40 & P. P. B. & M & Orelha E. & Amazōnia - Brasii \\
\hline 41 & J. M. C. & M & Braco $D$ & Amazônia _- Brasil \\
\hline 42 & & M & Pé E., braço D. e Nariz & SURINAM \\
\hline 43 & & M & Mãos & SURINAM \\
\hline 44 & & M & Antebraço $\mathrm{E}$. & SURINAM \\
\hline 45 & & $\mathrm{M}$ & Perna E. & SURINAM \\
\hline 46 & & M & Pé D. & SURINAM \\
\hline 47 & & M & Braco $D$. & SURINAM \\
\hline 48 & & M & Pé D. & SURINAM \\
\hline 49 & & M & Māo E. & Amazônia - Brasil \\
\hline 50 & A. C. $M$. & M & Perna D. & Amazonia - Brasil \\
\hline 51 & M. S. P. & M & Orelha D. & Amazōnia -... Brasil \\
\hline
\end{tabular}

AUTORES

ANOS

M. Morais 1962

M. Morais 1962

M. Morais 1962

M. Morais 1962

M. Morais \& W. Oliveira 1962

M. Morais \& W. Oliveira 1962

J. Michalany \& N. Lagonegro 1963

in Emmons \& Cols. - Medical Micology / J. P. 1963 Wiersema \& P. L. Niemel

in Emmons \& Cols. -.. Medical Micology / J. P. 1963/1965 Wiersema \& P. L. Niemel

in Emmons \& Cols. - Medical Micology / J.P. 1963 Wiersema \& P. L. Niemel

in Emnons \& Cols. -- Medical Micology / J. P. 1963 Wiersema \& P. L. Niemel

in Emmons \& Cols. - Medical Micology / J. P. 1963 Wiersema \& P. L. Niemel

in Emmons \& Cols. -.. Medical Micology / J. P. 1963 Wiersema \& P. L. Niemel

in Emmons \& Cols. Medical Micology / J P. 1963 Wiersema \& P. L. Niemel

$J$. Rodrigues Silva

Domingos Silva

Domingos Silva
1964

1965

1965 


\begin{tabular}{|c|c|}
\hline CASO & PACIEN \\
\hline No & TES \\
\hline & \\
\hline $\begin{array}{l}52 \\
53\end{array}$ & $\begin{array}{l}\mathrm{M} . \mathrm{X} \cdot \mathrm{A} . \\
\mathrm{R} . \mathrm{T}\end{array}$ \\
\hline 54 & F. C. C. \\
\hline 55 & M. A. R. \\
\hline 56 & P. G. N. \\
\hline 57 & D. R. B. \\
\hline 58 & \\
\hline 59 & \\
\hline 60 & A. I. C. \\
\hline 61 & A. C. \\
\hline 62 & $\mathrm{Tu}$ \\
\hline 63 & $\mathrm{Co}$ \\
\hline 64 & $\mathrm{Ta}$ \\
\hline 65 & $\mathrm{Mu}$ \\
\hline 66 & Ur \\
\hline 67 & Mo \\
\hline 68 & $\mathrm{Ma}$ \\
\hline
\end{tabular}

M. X. A.

M

Mão E., perna D.

Amazônia - Brasil

Amazônia - Brasil

Amazônia - Brasil

Amazônia - Brasil

Amazônia - Brasil

Amazônia - Brasil

Goiás - Brasil

COLÔMBIA

VENEZUELA

VENEZUELA

Amazônia Matogrossense - Brasil

Amazônia Matogrossense P. A. Machado / R. G. Baruzzi \& Cols. - Brasil.

Amazônia Matogrossense: P. A. Machacio / R. G. Baruzzi \& Cols. - Brasil

Amazônia Matogrossense P. A. Machado/R. G. Baruzzi \& Cols. - Brasil

Amazônia Matogrossense P. A. Machado/ R. G. Baruzzi \& Cols. - Brasil

Amazônia Matogrossense P. A. Machado/R. G. Baruzzi \& Cols. - Brasil

Amazônia Matogrossense P. A. Machado / R. G. Baruzzi \& Cols. - Brasil
Domingos Silva $\quad 1965$

Domingos Silva 1965

Domingos Silva

W. Barbosa \& J. Doles 1965

F. Battistine, S. G. Joger \& O. Perfetti 1966

1966

$1966 / 1967$

$1966 / 1967$

$1966 / 1967$

$1966 / 1967$

$1966 / 1967$

$1966 / 1967$

$1966 / 1967$ 


$$
1931 \ldots 1971
$$

\section{CASO PACIEN-}

NO TES

69

$\mathrm{C}_{\mathrm{O}}$

70

$\mathrm{Ce}$

71

72

73

74

75

76

77

78

79

80

81

82

83

84

85
R. M. M.

J. A. S.

F. B. S.

F. X. E

D. C. S.

A. J. M

I. P. C.

L. C. N.

SEXO

Ombro D.

Disseminada

Hemiface $\mathrm{E}$

Orelha D.

Nariz

Reg. peitoral D

Orelha E.

Perna E.

Mão D.

Braco E., braco D

Antebraço $\mathrm{E}$

Coxa D.

Pernas, antebraco D.

Orelha D.

Lábio superior

Nariz
Amazônia Matogrossense Brasil

Amazônia Matogrossense P. A. Machado / R. G. Baruzzi \& Cols. -... Brasil

Amazônia Matogrossense Brasil

Amazônia - - Brasil

Amazônia -- Brasil

Amazônia - Brasi

Amazónia -. Brasi

Amazônia - Brasil

GUIANA FRANCESA

Amazonia -... Brasi]

Amazônia - Brasil

Amazônia - Brasi

Amazonia .... Brasil

Amazônia - - Brasil

Amazônia -... Brasil

Anjazônia -- Brasil

Amazônia -- Brasil
P. A. Machado

J. Monteiro Leite

J. Monteiro Leite

$J$. Monteiro Leite

$J$. Monteiro Leite

J. Monteir L Lite

R. Pradinaud \& Cols.

L. Braga Dias, M. Sampaio, Domingos Silva

L. Braga Dias, M. Sampaio, Domingos Silva

Domingos Silva

Domingos Silva

Domingos Silva

Domingos Silva

Antonio P. Loureiro, A. C. Brito, Domingos Silva

A. Loureiro
$1966 / 1967$

$1966 / 1967$

1966

1967

1967

1967

1967

1967

1969

1970

1970

1970

1970

1970

1970

1970

1971 


$$
\begin{aligned}
& \text { MCOEE DE LOBO }
\end{aligned}
$$

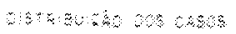

$$
\begin{aligned}
& \text { กิล }
\end{aligned}
$$

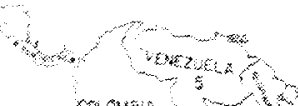

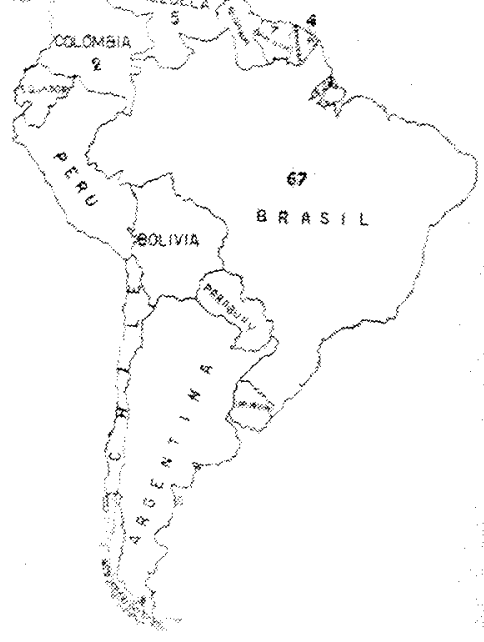

Fì. 1 - Distribuicão geografica da micose de Lobo 1931 - 1971.

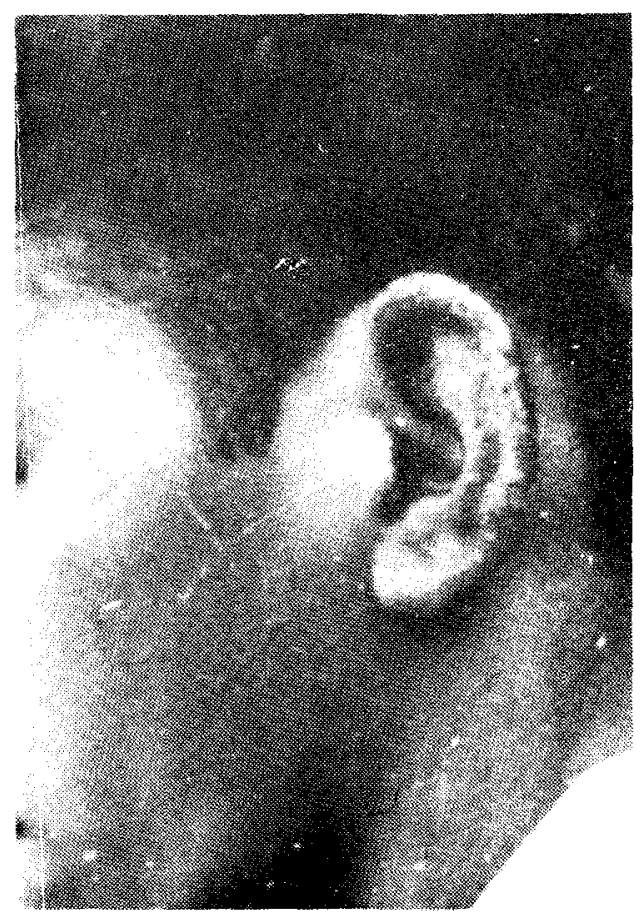

Fig. 3 - Forma clinica inicialmente infiltrativa.

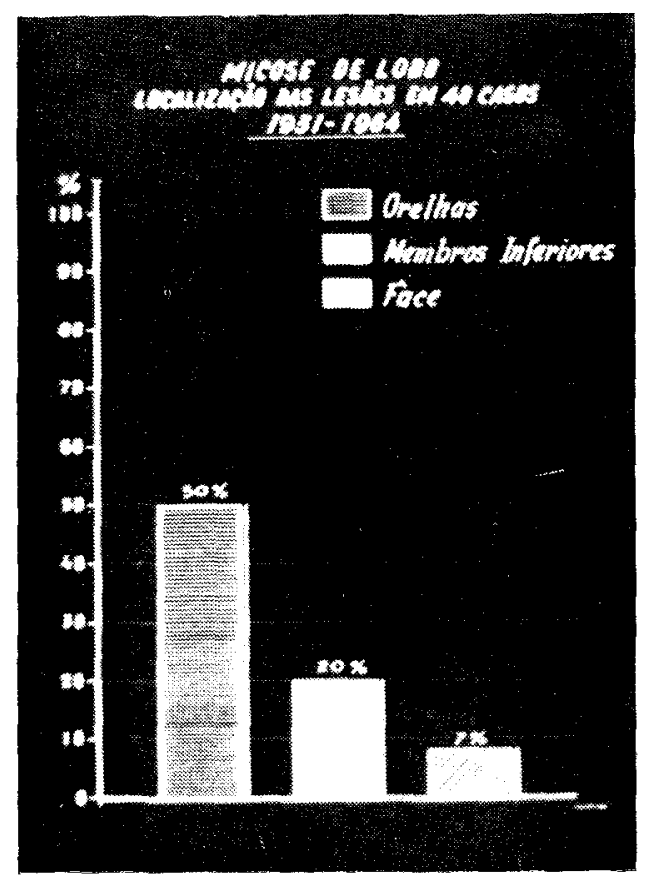

Fig. 2 -Localizaçảo da micose por áreas preferenciais.

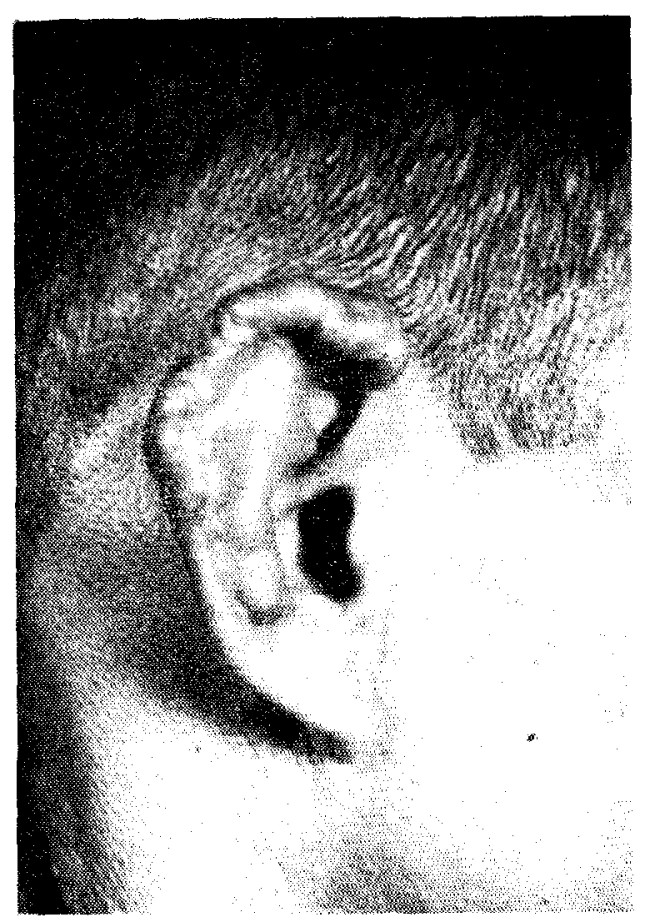

Fig. 4 - Forma infiltrativa onde se nota a passazem para o tipo queloidiano. 


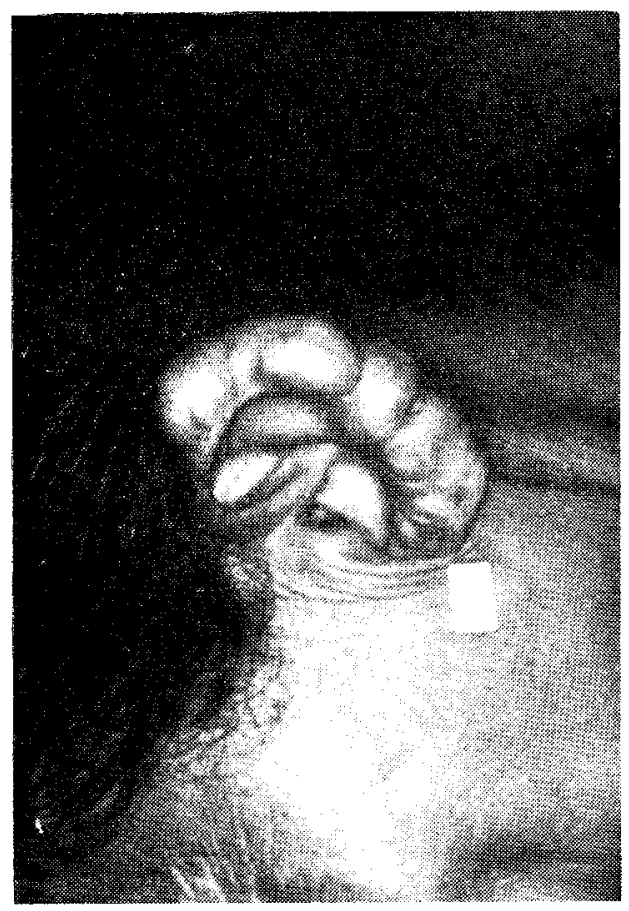

Fis. 5 -- Forma queloidiana tipica com locallzaçáo preferencial na orelba.

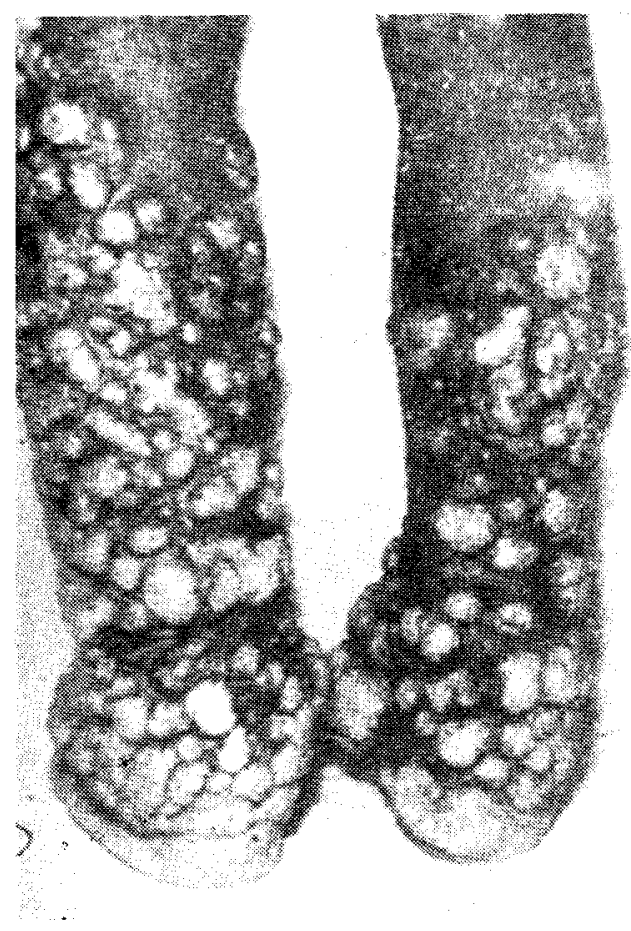

Fig. 6 -... Forma queloidiana extensa.

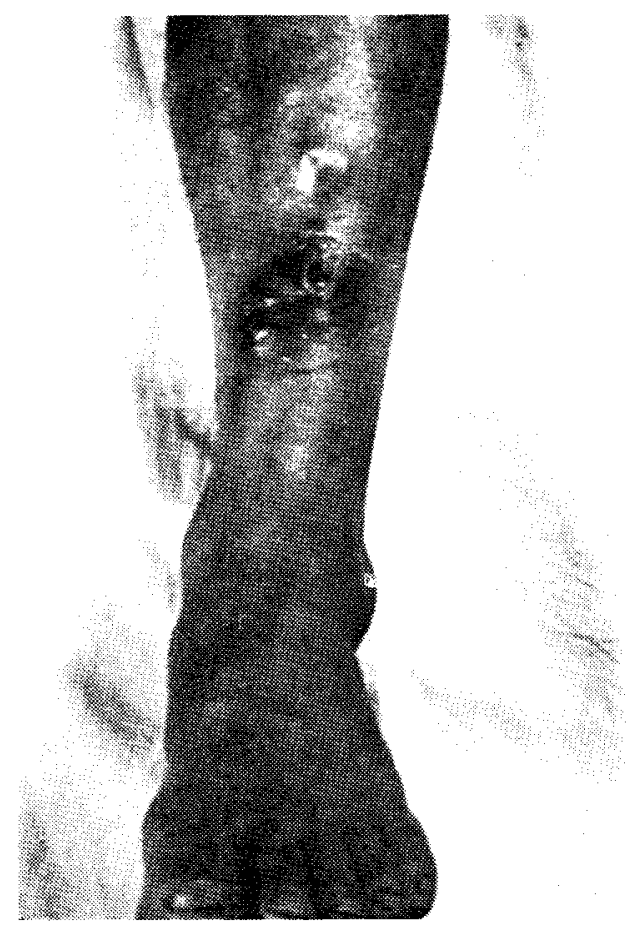

Fig. 7 - Tipo nodular ulcerado; acima da lesão uma goma ainda integra. 


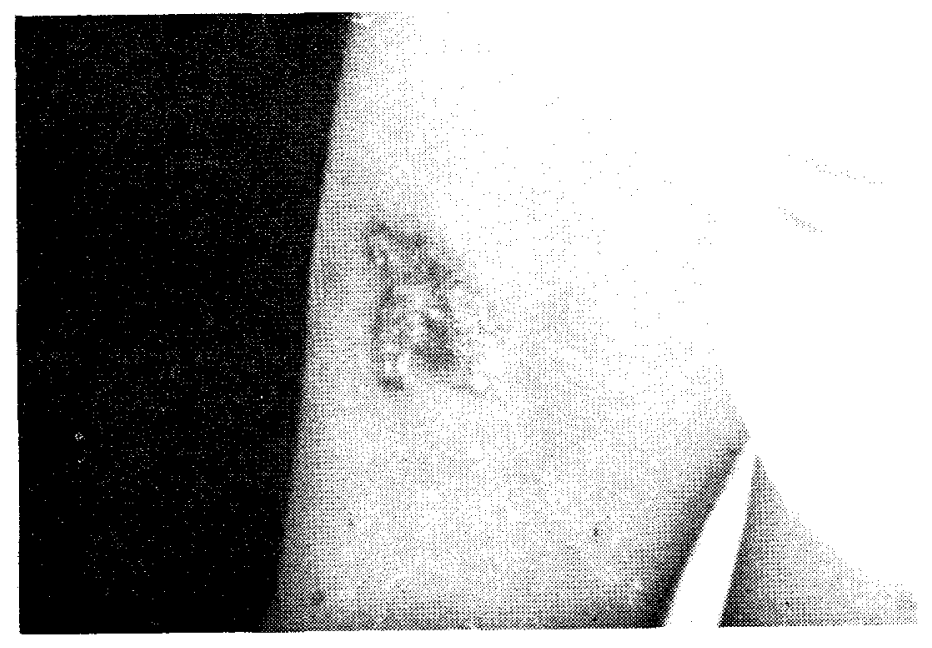

Fiv. 8 - Lesão gomosa tipica.

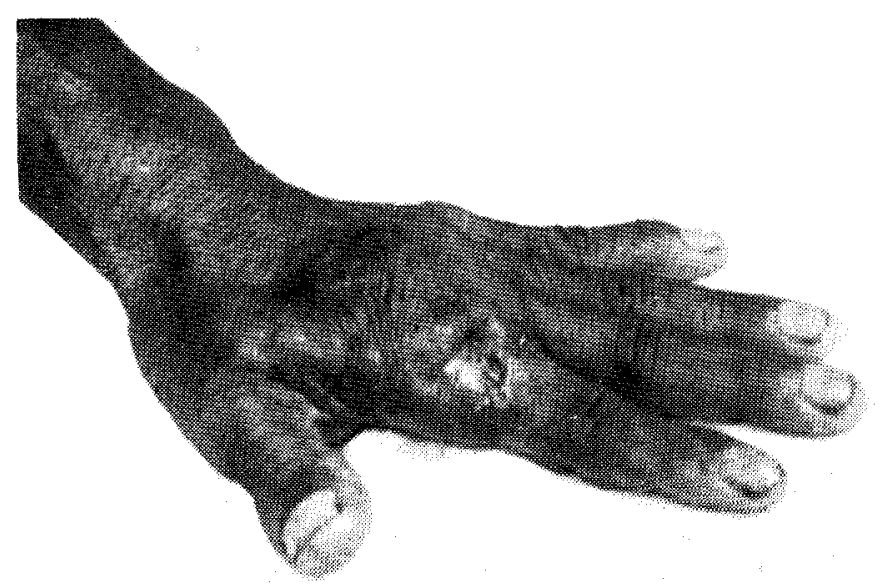

Fig. 9-Lesão ulcerosa franca. 


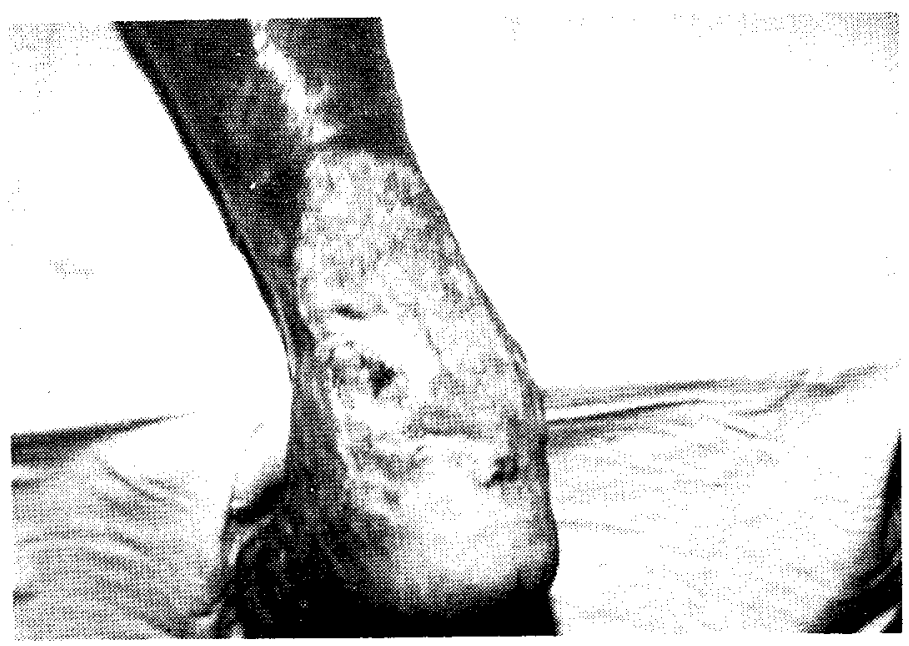

Fig. 1.0 - Lesāo ulcerosa típica

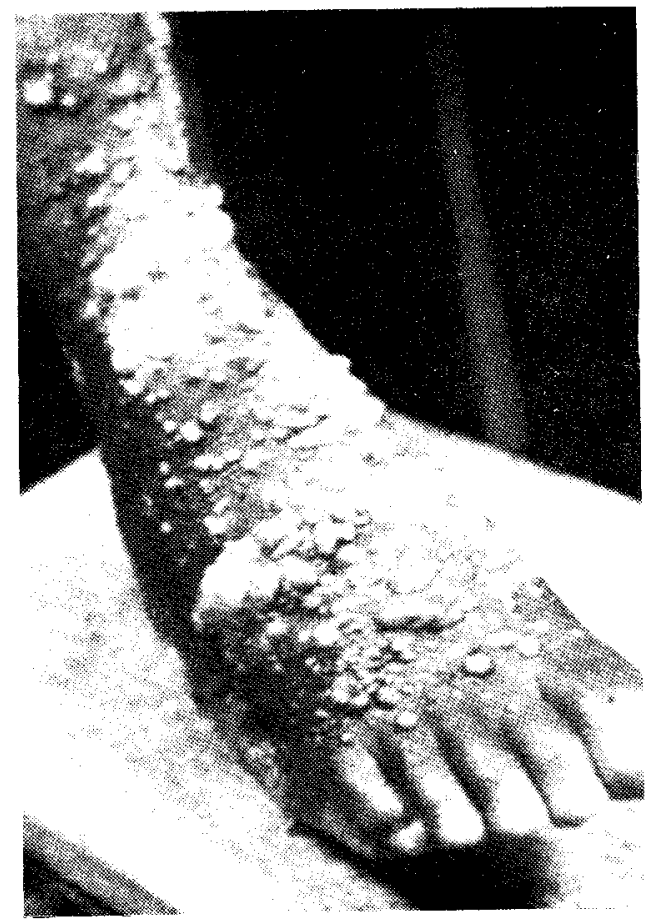

Fìg. 11 - Lesāo verructforme

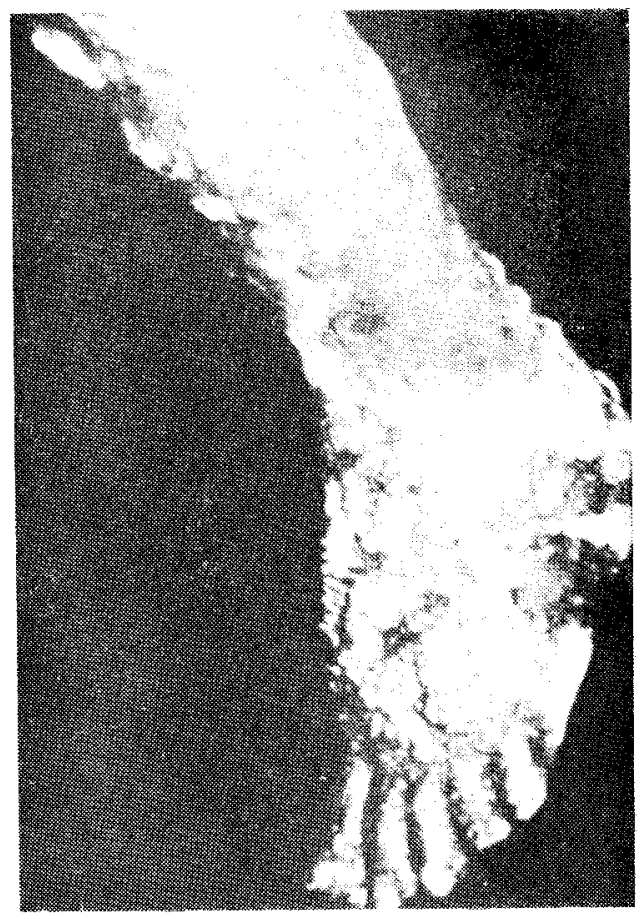

Fig. 12 - Lesão vegetante verrucosa 


\section{SUMMARY}

The author studios Jorge Lobo's mycosis, conidering the epidemistogial and clinical aspects of the disease.

In the author's coinion, the disease must not be called cheloidal biastomycosis. as, in his experience, ive cinical forms can be detected: infitrative, chelcilfonm, gomous, ilcerative and verrucous.

\section{PDEFTENOIAS BIBLIOGRAFTCAS}

1. AZDVEDO P. C. .... Algumas consideracoes sobre a Nioose de orge Lobo. Tese Fac Med. Univ. Para IErasil. Ed. Rev. Veterith, 1949

2. BAREOSA E. DOIES, - Blastomicose queloidiome idcenca de Jorea Lobol. Aprosentesád 10 caso encontrado no Fstado de Goias. Pev Goiana Med. 11: $11-20,1966$

3. BARUZZI, F G, DANDFFATMA JP. C.; CARUALAL S. RAMOE, O. ¿. $\&$ FONTES, P. L. - Cunrêcia da Blastcmicose quelcidiana ontre indios uaiabie Re. Inst. Med. Trop. Sâo Faulo 9: 135-142, 1967

4. BATTISTINI F.: JOVER, $\mathrm{s}$ G. $z$ PERFETTI. O. - Dos casos de blastomicosis oueloideana o erfermedad de Jcrge Lobo. Dermit. Venez. 5: 3036, 1965

5. BORELLI. D - Infomação psssoal.

6. CAMPO - AACHEN, I. - Nota pre via sobre el primer caso ce Enfermedad de Jorge Lobo o blastomicosis queloidiana en Venezuela. Derm. Venez. 1: $118-121,1957$

7. CONVIT, J. \& ESCUDER, R. - Blastomicose queloidiana o Enfermedad de Jorge Lobo. Derm. Venez., 2: 115120,1958 .

8. DIAS, L. B.; SAMPAIO, M. M. \& SILVA, D. - Jorge Lobo's Disease. Observations on its epidemiolcoy and some unusual morphological forms of the fungus. Rev. Inst. Med. Trop. $\mathrm{s}$. Faulo, 12: 8-15, 1970

9. FONTAN, R. - Primier cas de maladie de Lobo iblastomycose chéloidienne) en Guyane Francaise. Arch. Inst. Pasteur G. Franc. 46: 1-2, 1960.

10. FRAGA, S. \& MIRANDA, J. L. - Keloidal Blastomycosis (Jorge Lobo's Disease,. An. XIII Congressus Internationalis Dermatologiae, vol. 2: $830-$ $831,1968$.

11. HENAO PELAYO, C. - Blastomicosis queloideana. Rev. lat. amer. Anat. Path. 2: $139-143,1958$
12. HEREFRA, J. M. -- Parnoonctioides bresilienses ostudio del prinuer caso coservado al Panamé de blestomicosis sulamericana en sua foma cutanea gencialzada o srifermedad de Jorge Lcho y propuesta de una variante tecnica para la impresnación argentica del parasito Arch Med. Fanameñcs IV: 200-219, 1055.

13. LEITE, y M - Doenca de Jorge Lcoo. Tese Fac Mcd Univ Para Brasil, Grat. Rev. Véerinaria. 1954

14. LEITE, J M - Doenca de Jorge Lobo i Estido clinico patulogico, con apresentação de 5 cascs). Atas Simp. Biota Amaz. vol. 6: 161-176, 1967

15. LOUREIRO, A, A.; BRFTO, A. C \& SILVA. D. - Micose de Jorge Lobo de localizacão insolita. Apresentado ao XXVII Congr. Derm. Goiás, 1970.

16. MACHADO, P. A. - Contribuiçào para o estuda da epidemiologia da blastomicose queloidiforme de Jorge Lobo. Apresentado ao XVI Congresso Brasileiro de Higiene. Curitiba, Brasil, 1969

17. MORAIS. M. P. - Blastomicose tipo Jorge Lobo (seis novos casos encontrados no Estado do Amazcnas, Brasil). Rev. Inst. Med. Trop. São Paulo. 4: $187-197,1962$

18. PRADINAUD, R.; JOLY, F.; BASSET M.; MASSET, A. \& GRCSSHANS, E. - Les chromomycoses et la maladie de Jorge Lobo en Guyane Francaise Bull. Soc. Path. Exot. 62, 1054-1063 1969

19. REYES. O.; G O I H A N N. M \& GOLDSTEIN, C. - Blastomicosis queloidcana o Enfermedad de Jorge Lobo comunicación previa sobre un caso observado). Derm. Venez. IV: $\mathbf{2 1 5}-\mathbf{3 5 5}$. 1961 .

20. ROMERO, A. \& TREJOS, A. - Contribuicão ao estudo das blastomicoses em Costa Rica. Rev, Biol. Trop., 1 : 63-81. 1953 . 
21. SILVA, D - Sur un nouveau cas de la Mycose de Jcrge Lobo. Arch. belges dermat, 13: 26 35, 1957.

22. BIIVA. D.: FERREIRA, J. L. S.: BRITO. A. C. \& PINTO, N. - Consideracoes preliminares sobre dois novos caSus a Micose de Jorge Lobo. Rev. Fac. Med. Univ. Ceara (Brasil). 3: 19, 1963.

23. SILVA, D - Oito casos novos da $\mathrm{Mi}$ cose queloidiforme de Jorge Lobo. An. bras. derm. sit. 40: 307-326, 1965

24. SILVA, D - Clinical and epidemiological studies of Jorge Lobo's Mycosis. Proceedings of 2 th Int. Cong. Trop. Dermatology. Japan, 1970
25. SILVERIE, R.; RAVISSE, P.: VILAR, J. P. \& MOULINS, C. - La blastomycose chéloidienne ou maladie de Jorge Lobo en Guyane Francaise. Bull. Soc. Path. Exot. 56: 29-35, 1963

26. VILIEGAS, M. R. - Enfermedad de Jorge Lobo iblastomicosis queloidiana). Presentación de un nuevo caso Colombiano. Micopath. IDen Haag: 25: $373-380,1965$.

27. WIERSEMA, J. P. \& NIEMEL, P. $L$ - Lobo's disease in Surinam patients. Trop. Geog. Med. 17: 89-111, 1965. 